\title{
A GENERALIZATION OF THE DISCRETE HARDY'S INEQUALITY
}

\section{SUI SUN CHENG AND REUIH FEN LU}

In this note we are concerned with a generalization of the well known Hardy's inequality for series [1, pp. 239-241] (in the sequel, $p$ shall denote a real number greater than 1):

Theorem 1. If $p>1, a_{k} \geq 0$ for $k=1,2,3, \cdots$ and $A_{k}=a_{1}+a_{2}+a_{3}+$ $\ldots+a_{k}$, then

$$
\sum_{k=1}^{n}\left[\frac{A_{k}}{k}\right]^{p}<\left[\frac{p}{p-1}\right]^{p} \sum_{k=1}^{n} a_{k}^{p},
$$

unless $a_{k}=0$ for $k=1,2, \cdots, n$; and suppose further that

$$
\sum_{k=1}^{\infty} a_{k}^{p}<\infty
$$

then

$$
\sum_{k=1}^{\infty}\left[\frac{A_{k}}{k}\right]^{p}<\left[\frac{p}{p-1}\right]^{p} \sum_{k=1}^{\infty} a_{k}^{p},
$$

unless $a_{k}=0$ for $k=1,2,3, \cdots$. The constant $(p /(p-1))^{p}$ is the best possible.

We shall view inequalities (1) and (2) as necessary conditions for the existence of positive nondecreasing solutions of a nonlinear recurrence relation. To be more precise, consider the following recurrence relation of the form

$$
\Delta\left(r_{k-1}\left(\Delta y_{k-1}\right)^{p-1}\right)+s_{k-1} y_{k}^{p-1} \leq 0, \quad k=1,2, \cdots, n,
$$

Received October 15, 1992. 
where $p>1, r_{k}>0$ for $k=0,1, \cdots, n$ and $s_{k} \in R$ for $k=0,1, \cdots, n-1$. A solution of (3) is a real sequence $\left\{y_{k}\right\}_{k=0}^{n+1}$ which satisfies (3).

Given a fixed pair of real numbers $\alpha \geq-1$ and $\beta \leq-1$, a real sequene $y=\left\{y_{k}\right\}_{k=0}^{n+1}$ is said to be admissible if it satisfies the following conditions:

$$
\begin{aligned}
& y_{0}+\alpha y_{1}=0, \\
& y_{n+1}+\beta y_{n}=0, \\
& y_{k} \geq 0 \text { for } 1 \leq k \leq n, \\
& \Delta y_{k} \geq 0 \text { for } 1 \leq k \leq n-1 .
\end{aligned}
$$

Note that the condition $\alpha \geq-1$ implies that $\Delta y_{0} \geq 0$ and the condition $\beta \leq-1$ implies that $\Delta y_{n} \geq 0$.

For any admissible sequence $v=\left\{v_{k}\right\}_{k=0}^{n+1}$, define the functional

$$
J[v]=r_{0}(1+\alpha)^{p-1} v_{1}^{p}+\sum_{k=1}^{n-1} r_{k}\left(\Delta v_{k}\right)^{p}-\sum_{k=1}^{n} s_{k-1} v_{k}^{p}-r_{n}(-1-\beta)^{p-1} v_{n}^{p} .
$$

We shall need the following well known result,

Lemma 1. If $x, y \geq 0$ and $p>1$, then $p x^{p-1}(x-y) \geq x^{p}-y^{p}$, equality holds if and only if $x=y$. If $b \geq 0, a+b \geq 0$ and $p>1$, then $(a+b)^{p} \geq p a b^{p-1}+b^{p}$, equality holds if and only if $a=0$.

By means of this Lemma, we can show the following necessary condition for the existence of a nonnegative nondecreasing solution of (3).

Theorem 2. Suppose $y=\left\{y_{k}\right\}_{0}^{n+1}$ is an admissible solution of (3) such that $y_{k}>0$ for $1 \leq k \leq n$. Then for any admissible sequence $u=\left\{u_{k}\right\}_{0}^{n+1}$, we have $J[u] \geq 0$, equality holds if and only if one of the following conditions holds: (1) $u \equiv 0$, or (2) $y$ is a solution of

$$
\Delta\left(r_{k-1}\left(\Delta y_{k-1}\right)^{p-1}\right)+s_{k-1} y_{k}^{p-1}=0, \quad k=1,2, \cdots, n,
$$

and $u$ is a constant but non-zero multiple of $y$. 
Proof. Let $z_{k}=u_{k} / y_{k}$ for $1 \leq k \leq n$. Then $z_{k} \geq 0$ and $u_{k}=z_{k} y_{k}$ for $1 \leq k \leq n$. Since $u$ is admissible, thus

$$
0 \leq \Delta u_{k}=y_{k} \Delta z_{k}+z_{k+1} \Delta y_{k}
$$

for $k=1,2, \cdots, n-1$. It follows from the admissibility of $y$ and Lemma 1 that

$$
\begin{aligned}
\left(\Delta u_{k}\right)^{p} & =\left(y_{k} \Delta z_{k}+z_{k+1} \Delta y_{k}\right)^{p} \\
& \geq p y_{k} \Delta z_{k}\left(z_{k+1} \Delta y_{k}\right)^{p-1}+\left(z_{k+1} \Delta y_{k}\right)^{p} \\
& =y_{k}\left\{p z_{k+1}^{p-1} \Delta z_{k}\right\}\left(\Delta y_{k}\right)^{p-1}+\left(z_{k+1} \Delta y_{k}\right)^{p} \\
& \geq y_{k}\left(\Delta z_{k}^{p}\right)\left(\Delta y_{k}\right)^{p-1}+\left(z_{k+1} \Delta y_{k}\right)^{p}
\end{aligned}
$$

for $k=1, \cdots, n-1$, where, by Lemma 1 , the first inequality is an equality if and only if

$$
y_{k} \Delta z_{k}=0, \quad k=1, \cdots, n-1
$$

and the second is an equality if and only if

$$
\Delta z_{k}=0, \quad k=1, \cdots, n-1 .
$$

As a consequence, we have

$$
\sum_{k=1}^{n-1} r_{k}\left(\Delta u_{k}\right)^{p} \geq \sum_{k=1}^{n-1} r_{k} y_{k}\left(\Delta z_{k}^{p}\right)\left(\Delta y_{k}\right)^{p-1}+\sum_{k=1}^{n-1} r_{k}\left(z_{k+1} \Delta y_{k}\right)^{p}
$$

and equality holds if and only if $\Delta z_{k}=0$ for $k=1,2, \cdots, n-1$ (since $y_{k}>0$ for $1 \leq k \leq n-1)$. The conditions that $\Delta z_{k}=0$ for $k=1,2, \cdots, n-1$ is equivalent to $u=c y$. Indeed, if $\Delta z_{k}=0$ for $k=1, \cdots, n-1$, then $u_{k}=c y_{k}$ for $1 \leq k \leq n$. But then $u_{0}=-\alpha u_{1}=-\alpha c y_{1}=c y_{0}$ and similarly that $u_{n+1}=c y_{n+1}$. The converse is clearly true.

Note that the first sum on the right hand side of (8), if we apply the Abel's 
transformation, changes to

$$
\begin{aligned}
\sum_{k=1}^{n-1} \Delta\left(r_{k} y_{k}\left(\Delta y_{k}\right)^{p-1} z_{k}^{p}\right]-\sum_{k=1}^{n-1} z_{k+1}^{p} \Delta\left[y_{k} r_{k}\left(\Delta y_{k}\right)^{p-1}\right] \\
=r_{n}\left[\frac{\Delta y_{n}}{y_{n}}\right]^{p-1} u_{n}^{p}-r_{1}\left(\Delta y_{1}\right)^{p-1} y_{1} z_{1}^{p} \\
\quad-\sum_{k=1}^{n-1} z_{k+1}^{p} y_{k+1} \Delta\left[r_{k}\left(\Delta y_{k}\right)^{p-1}\right]-\sum_{k=1}^{n-1} r_{k}\left(z_{k+1} \Delta y_{k}\right)^{p} \\
=r_{n}(-1-\beta)^{p-1} u_{n}^{p}-\left\{y_{1} z_{1}^{p} \Delta\left[\left(r_{0}\left(\Delta y_{0}\right)^{p-1}\right]+r_{0}(1+\alpha)^{p-1} u_{1}^{p}\right\}\right. \\
\quad-\sum_{k=1}^{n-1} z_{k+1}^{p} y_{k+1} \Delta\left[r_{k}\left(\Delta y_{k}\right)^{p-1}\right]-\sum_{k=1}^{n-1} r_{k}\left(z_{k+1} \Delta y_{k}\right)^{p}
\end{aligned}
$$

where we have used (4) and (5) in obtaining the last equality.

Thus

$$
\begin{gathered}
\sum_{k=1}^{n-1} r_{k}\left(\Delta u_{k}\right)^{p} \geq-\sum_{k=0}^{n-1} z_{k+1}^{p} y_{k+1} \Delta\left[r_{k}\left(\Delta y_{k}\right)^{p-1}\right]+r_{n}(-1-\beta)^{p-1} u_{n}^{p} \\
-r_{0}(1+\alpha)^{p-1} u_{1}^{p}
\end{gathered}
$$

where equality holds if and only if $u_{k}=c y_{k}$ for $0 \leq k \leq n+1$. Since by (3),

$$
-\sum_{k=0}^{n-1} z_{k+1}^{p} y_{k+1} \Delta\left[r_{k}\left(\Delta y_{k}\right)^{p-1}\right] \geq \sum_{k=0}^{n-1} z_{k+1}^{p} y_{k+1} s_{k} y_{k+1}^{p-1}
$$

$J[u] \geq 0$, and equality holds if and only if, $u_{k}=c y_{k}\left(\right.$ i.e. $z_{k}=c$ ) for $0 \leq k \leq n+1$ and

$$
\sum_{k=0}^{n-1} z_{k+1}^{p} y_{k+1}\left\{\Delta\left[r_{k}\left(\Delta y_{k}\right)^{p-1}\right]+s_{k} y_{k+1}^{p-1}\right\}=0
$$

if and only if, either $c=0$, or, $c \neq 0$ and $(3)^{\prime}$ holds. The proof is complete.

Similarly, consider the following recurrence relation of the form

$$
\Delta\left(r_{k-1}\left(\Delta y_{k-1}\right)^{p-1}\right)+s_{k-1} y_{k}^{p-1} \leq 0, \quad k=1,2, \cdots
$$

where $p>1, r_{k}>0$ for $k \geq 0$, and $s_{k} \in R$ for $k \geq 0$. A solution of (9) is a real sequence $\left\{y_{k}\right\}_{k=0}^{\infty}$ which satisfies (9). 
Given a fixed real numbers $\alpha \geq-1$, a real sequence $y=\left\{y_{k}\right\}_{k=0}^{\infty}$ is said to be admissible if it satisfies the following conditions:

$$
\begin{aligned}
& y_{0}+\alpha y_{1}=0 \\
& y_{k} \geq 0 \text { for } \quad k \geq 1 \\
& \Delta y_{k} \geq 0 \text { for } \quad k \geq 1
\end{aligned}
$$

For any admissible sequence $v=\left\{v_{k}\right\}_{k=0}^{\infty}$, define the functional

$$
H[v]=r_{0}(1+\alpha)^{p-1} v_{1}^{p}+\sum_{k=1}^{\infty} r_{k}\left(\Delta v_{k}\right)^{p}-\sum_{k=1}^{\infty} s_{k-1} v_{k}^{p} .
$$

Theorem 3. Suppose $y=\left\{y_{k}\right\}_{0}^{\infty}$ is an admissible solution of (9) such that $y_{k}>0$ for $k \geq 1$. Then for any admissible sequence $u=\left\{u_{k}\right\}_{0}^{\infty}$ such that

$$
\sum_{k=1}^{\infty} r_{k}\left(\Delta u_{k}\right)^{p}<\infty
$$

we have $H[u] \geq 0$, equality holds if and only if one of the following conditions holds: (1) $u \equiv 0$; or (2) $y$ is a solution of

$$
\Delta\left(r_{k-1}\left(\Delta y_{k-1}\right)^{p-1}\right)+s_{k-1} y_{k}^{p-1}=0, \quad k=1,2, \cdots
$$

and $u$ is a constant but non-zero multiple of $y$.

The proof is similar to that of Theorem 2. Let $z_{k}=u_{k} / y_{k}$ for $k \geq 1$. Then

$$
\sum_{k=1}^{\infty} r_{k}\left(\Delta u_{k}\right)^{p} \geq \sum_{k=1}^{\infty} r_{k} y_{k}\left(\Delta z_{k}^{p}\right)\left(\Delta y_{k}\right)^{p-1}+\sum_{k=1}^{\infty} r_{k}\left(z_{k+1} \Delta y_{k}\right)^{p}
$$

and equality holds if and only if $u_{k}=c y_{k}$ for $k \geq 0$. Also,

$$
\begin{aligned}
& \sum_{k=1}^{\infty} r_{k} y_{k}\left(\Delta z_{k}^{p}\right)\left(\Delta y_{k}\right)^{p-1} \\
= & -r_{0}(1+\alpha)^{p-1} u_{1}^{p}-\sum_{k=0}^{\infty} z_{k+1}^{p} y_{k+1} \Delta\left[r_{k}\left(\Delta y_{k}\right)^{p-1}\right]-\sum_{k=1}^{\infty} r_{k}\left(z_{k+1} \Delta y_{k}\right)^{p} .
\end{aligned}
$$


Thus

$$
\sum_{k=1}^{\infty} r_{k}\left(\Delta u_{k}\right)^{p} \geq \sum_{k=1}^{\infty} s_{k-1} u_{k}^{p}-r_{0}(1+\alpha)^{p-1} u_{1}^{p}
$$

where equality holds if and only if, $u=c y$ and

$$
\sum_{k=0}^{\infty} z_{k+1}^{p} y_{k+1}\left\{\Delta\left[r_{k}\left(\Delta y_{k}\right)^{p-1}\right]+s_{k} y_{k+1}^{p-1}\right\}=0
$$

if and only if either $c=0$, or $c \neq 0$ and $(9)^{\prime}$ holds. The proof is complete.

We assert that Theorem 1 follows from Theorem 2 and 3 . To see this, we first show that the following recurrence relation

$$
\Delta\left(\Delta y_{k-1}\right)^{p-1}+\left[\frac{p-1}{p}\right]^{p}\left[\frac{1}{k}\right]^{p} y_{k}^{p-1}<0, \quad k=1,2, \cdots,
$$

has a solution $w=\left\{w_{k}\right\}_{0}^{\infty}$ such that $w_{0}=0, w_{1}=1, w_{k}>0$ and $\Delta w_{k}>0$ for $k \geq 1$, and $\Delta w_{k} / w_{k} \rightarrow 0$ as $k \rightarrow \infty$.

Indeed, let $w_{0}=0, w_{k}=k^{(p-1) / p}$ for $k \geq 1$. Then by the mean value theorem,

$$
\Delta w_{k}=(k+1)^{(p-1) / p_{-k}(p-1) / p}=\left[\frac{p-1}{p}\right] \mu_{k}^{-1 / p}, \quad k<\mu_{k}<k+1
$$

so that

$$
\Delta w_{k}<\left[\frac{p-1}{p}\right] k^{-1 / p}
$$

Similarly,

$$
\Delta w_{k-1}=\left[\frac{p-1}{p}\right] \mu_{k-1}^{-1 / p}, \quad k-1<\mu_{k-1}<k .
$$

Thus

$$
\begin{aligned}
\Delta\left(\Delta w_{k-1}\right)^{p-1} & <\left[\frac{p-1}{p}\right]^{p-1}\left[k^{-(p-1) / p}-\mu_{k-1}^{-(p-1) / p}\right] \\
& =-\left[\frac{p-1}{p}\right]^{p} \mu^{(1-2 p) / p}, \quad k-1<\mu<k .
\end{aligned}
$$

It follows that

$$
\begin{aligned}
& \Delta\left(\Delta w_{k-1}\right)^{p-1}+\left[\frac{p-1}{p}\right]^{p}\left[\frac{1}{k}\right]^{p} w_{k}^{p-1} \\
< & -\left[\frac{p-1}{p}\right]^{p} \mu^{(1-2 p) / p}+\left[\frac{p-1}{p}\right]^{p}\left[\frac{1}{k}\right]^{p} k^{(p-1)^{2} / p} \\
= & {\left[\frac{p-1}{p}\right]^{p}\left[k^{(1-2 p) / p}-\mu^{(1-2 p) / p}\right]<0 }
\end{aligned}
$$


as desired. The fact that $w_{k+1} / w_{k} \rightarrow 1$ is clear. Our assertion is proved.

Now let $a_{k} \geq 0$ for $k=1,2, \cdots, n$. Let $A_{0}=0, A_{1}=a_{1}, A_{2}=a_{1}+a_{2}, \ldots$, $A_{n}=a_{1}+\ldots+a_{n}$ and $A_{n+1}=-\beta_{n} A_{n}$ where $\beta_{n}=-w_{n+1} / w_{n}<-1$. Then $\left\{A_{k}\right\}_{0}^{n+1}$ is an admissible sequence with respect to $\alpha=0$ and $\beta=\beta_{n}$. Thus by Theorem 2,

$$
\sum_{k=1}^{n} a_{k}^{p} \geq \sum_{k=1}^{n}\left[\frac{p-1}{p}\right]^{p}\left[\frac{A_{k}}{k}\right]^{p}+\left[\frac{w_{n+1}}{w_{n}} 1\right]^{p-1} A_{n}^{p}
$$

where equality holds if and only if $A_{k}=0$ for $0 \leq k \leq n+1$. This implies

$$
\sum_{k=1}^{n} a_{k}^{p}>\sum_{k=1}^{n}\left[\frac{p-1}{p}\right]^{p}\left[\frac{A_{k}}{k}\right]^{p}
$$

unless $a_{k}=0$ for $1 \leq k \leq n$, which extends (1).

Similarly, let $a_{k} \geq 0$ for $k \geq 1, A_{0}=0, A_{k}=a_{1}+\ldots+a_{k}$ for $k \geq 1$. Then $\left\{A_{k}\right\}_{0}^{\infty}$ is an admissible sequence with respect to $\alpha=0$. If

$$
\sum_{n=1}^{\infty} a_{n}^{p}<\infty
$$

then by Theorem 3 ,

$$
\sum_{k=1}^{\infty} a_{k}^{p} \geq \sum_{k=1}^{\infty}\left[\frac{p-1}{p}\right]^{p}\left[\frac{A_{k}}{k}\right]^{p}
$$

where equality holds if and only if $A_{k}=0$ for $k \geq 0$. Thus (2) holds unless $a_{k}=0$ for $k \geq 1$.

\section{Acknowledgement}

The first author is supported by NSC of Republic of China.

\section{References}

[1] G. H. Hardy, J. E. Littlewood and G. Polya, "Inequalities", 2nd ediiion, Cambridge University Press, 1988.

Department of Mathematics, Tsing Hua University, Taiwan, Republic of China, and, Department of Electronic Engineering, Van Nung Institute of Technology, Taiwan, Republic of China. 